\title{
Vaccination Coverage Among Children Aged 19-35 Months — United States, 2015
}

\author{
Holly A. Hill, MD, PhD ${ }^{1}$; Laurie D. Elam-Evans, $\mathrm{PhD}^{1}$; David Yankey, MS, $\mathrm{MPH}^{1}$; James A. Singleton, $\mathrm{PhD}^{1}$; Vance Dietz, $\mathrm{MD}^{1}$
}

Sustained high coverage with recommended vaccinations among children has kept many vaccine-preventable diseases at low levels in the United States (1). To assess coverage with vaccinations recommended for children by age 2 years in the United States (2), CDC analyzed data collected by the 2015 National Immunization Survey (NIS) for children aged 19-35 months (born January 2012-May 2014). Overall, coverage did not change during 2014-2015. Coverage in 2015 was highest for $\geq 3$ doses of poliovirus vaccine $(93.7 \%), \geq 3$ doses of hepatitis B vaccine (HepB) $(92.6 \%), \geq 1$ dose of measles, mumps, and rubella vaccine (MMR) $(91.9 \%)$, and $\geq 1$ dose of varicella vaccine $(91.8 \%)$. The data were also examined for potential vaccination coverage differences by race/ethnicity, poverty status, and urbanicity. Although disparities were noted for each of these factors, the most striking differences were seen for poverty status. Children living below the federal poverty level* had lower coverage with most of the vaccinations assessed compared with children living at or above the poverty level; the largest disparities were for rotavirus vaccine $(66.8 \%$ versus $76.8 \%$ ), $\geq 4$ doses of pneumococcal conjugate vaccine (PCV) $(78.9 \%$ versus $87.2 \%)$, the full series of Haemophilus influenzae type b vaccine (Hib) (78.1\% versus $85.5 \%)$, and $\geq 4$ doses of diphtheria, tetanus, and acellular pertussis vaccine (DTaP) (80.2\% versus $87.1 \%)$. Although coverage was high in some groups, opportunities exist to continue to address disparities. Implementation of evidence-based interventions, including strategies to enhance access to vaccination services and systems strategies that can reduce missed opportunities, has the potential to increase vaccination coverage for children living below the poverty level and in rural areas (3).

NIS monitors vaccination coverage among children aged 19-35 months in the 50 states, the District of Columbia, selected local areas, and territories ${ }^{\dagger}$ using a random digit dialing (RDD) sample of landline and cellular telephone numbers. After identifying a household with at least one age-eligible

\footnotetext{
* Poverty level uses income and family size to categorize households into those 1) at or above the poverty level, and 2) below the poverty level. Poverty level was based on 2014 U.S. Census poverty thresholds (http://www.census.gov/ hhes/www/poverty/data/threshold).

$\dagger$ The local areas sampled separately for the 2015 NIS included areas that receive federal Section 317 immunization funds and are included in the NIS sample every year (Chicago, Illinois; New York, New York; Philadelphia County, Pennsylvania; Bexar County, Texas; and Houston, Texas) and two additional sample areas (El Paso County, Texas and Hidalgo County, Texas). The 2015 NIS was also conducted in Guam, Puerto Rico, and the U.S. Virgin Islands; these three territories were excluded from national coverage estimates.
}

child, a telephone interview is conducted to collect sociodemographic characteristics for all age-eligible children and request permission to contact the child's vaccination providers. If consent is given, a survey is mailed to each provider to request the child's vaccination history, including dates of receipt of vaccine doses. All coverage estimates are based on providerreported vaccination histories. Details regarding NIS methodology and weighting have been described previously. ${ }^{\S}$ For 2015 , national vaccination coverage estimates were based on a sample of 15,167 children with completed household interviews and adequate provider data. The Council of American Survey Research Organizations (CASRO) response rate was $34.9 \%$. Logistic regression was used to assess differences among racial/ethnic groups, controlling for poverty status, and to evaluate the potential interaction between poverty status and Metropolitan Statistical Area* (MSA) status (a measure of urbanicity). Statistical comparisons were made using t-tests on weighted data, taking into account the complex survey design. P-values $<0.05$ were considered statistically significant.

\section{National Vaccination Coverage}

Nationally, coverage did not change during 2014-2015 for the vaccinations assessed, and the percentage of children who received no vaccinations remained $<1 \%$ (Table 1 ). The Healthy People $2020^{\dagger \dagger}$ target of $90 \%$ coverage was met for four vaccines: 1$) \geq 3$ doses of poliovirus vaccine $(93.7 \%), 2) \geq 3$ doses of

\footnotetext{
$\$$ Further details regarding the statistical methodology of NIS are available in the NIS User's Guide 2014, which can be downloaded at http://www.cdc. gov/nchs/nis/data_files.htm.

The CASRO household response rate, calculated as the product of the resolution rate (percentage of the total telephone numbers called that were classified as nonworking, nonresidential, or residential), screening completion rate (percentage of known households that were successfully screened for the presence of age-eligible children), and the interview completion rate (percentage of households with one or more age-eligible children that completed the household survey) (http://www.casro.org). The CASRO response rate is equivalent to the American Association for Public Opinion Research (AAPOR) type 3 response rate (http://www.aapor.org/AAPOR Main/media/publications/Standard-Definitions20169theditionfinal.pdf). The 15,167 children with adequate provider data in the 2015 NIS represent $56.2 \%$ of children with completed household interviews.

** Metropolitan Statistical Areas have at least one urbanized area of 50,000 or more population, plus adjacent territory that has a high degree of social and economic integration with the core as measured by commuting ties. The Office of Management and Budget published the Standards for Delineating Metropolitan Statistical Areas in 2010; current definitions are based on an update published in February of 2013 (https://www.whitehouse.gov/sites/ default/files/omb/bulletins/2015/15-01.pdf).

${ }^{\dagger} \mathrm{https}$ //www.healthypeople.gov/2020/topics-objectives/topic/ immunization-and-infectious-diseases.
} 
TABLE 1. Estimated vaccination coverage among children aged 19-35 months, by selected vaccines and doses —National Immunization Survey, United States, 2011-2015*

\begin{tabular}{|c|c|c|c|c|c|}
\hline & 2011 & 2012 & 2013 & 2014 & 2015 \\
\hline Vaccine/Dose & $\%(95 \% \mathrm{Cl})$ & $\%(95 \% \mathrm{Cl})$ & $\%(95 \% \mathrm{Cl})$ & $\%(95 \% \mathrm{Cl})$ & $\%(95 \% \mathrm{Cl})$ \\
\hline \multicolumn{6}{|l|}{$\mathrm{DTaP}^{\dagger}$} \\
\hline$\geq 3$ doses & $95.5( \pm 0.5)$ & $94.3( \pm 0.7)^{* *}$ & $94.1( \pm 0.9)$ & $94.7( \pm 0.7)$ & $95.0( \pm 0.6)$ \\
\hline$\geq 4$ doses & $84.6( \pm 1.0)$ & $82.5( \pm 1.2)^{* *}$ & $83.1( \pm 1.3)$ & $84.2( \pm 1.2)$ & $84.6( \pm 1.1)$ \\
\hline Poliovirus ( $\geq 3$ doses) & $93.9( \pm 0.6)$ & $92.8( \pm 0.7)^{* *}$ & $92.7( \pm 1.0)$ & $93.3( \pm 0.8)$ & $93.7( \pm 0.6)$ \\
\hline MMR ( $\geq 1$ dose) & $91.6( \pm 0.8)$ & $90.8( \pm 0.8)$ & $91.9( \pm 0.9)$ & $91.5( \pm 0.9)$ & $91.9( \pm 0.8)$ \\
\hline \multicolumn{6}{|l|}{$\mathrm{Hib}^{\S}$} \\
\hline Primary series & $94.2( \pm 0.6)^{* *}$ & $93.3( \pm 0.7)$ & $93.7( \pm 0.9)$ & $93.3( \pm 0.8)$ & $94.3( \pm 0.6)$ \\
\hline Full series & $80.4( \pm 1.1)^{* *}$ & $80.9( \pm 1.2)$ & $82.0( \pm 1.3)$ & $82.0( \pm 1.3)$ & $82.7( \pm 1.1)$ \\
\hline \multicolumn{6}{|l|}{ НерB } \\
\hline$\geq 3$ doses & $91.1( \pm 0.7)$ & $89.7( \pm 0.9)^{* *}$ & $90.8( \pm 1.0)$ & $91.6( \pm 0.9)$ & $92.6( \pm 0.7)$ \\
\hline Birth dose & $68.6( \pm 1.3)^{* *}$ & $71.6( \pm 1.4)^{* *}$ & $74.2( \pm 1.4)^{* *}$ & $72.4( \pm 1.5)$ & $72.4( \pm 1.4)$ \\
\hline Varicella ( $\geq 1$ dose) & $90.8( \pm 0.7)$ & $90.2( \pm 0.8)$ & $91.2( \pm 0.9)$ & $91.0( \pm 0.9)$ & $91.8( \pm 0.8)$ \\
\hline \multicolumn{6}{|l|}{ PCV } \\
\hline$\geq 3$ doses & $93.6( \pm 0.6)^{* *}$ & $92.3( \pm 0.8)^{* *}$ & $92.4( \pm 1.0)$ & $92.6( \pm 0.8)$ & $93.3( \pm 0.7)$ \\
\hline$\geq 4$ doses & $84.4( \pm 1.0)$ & $81.9( \pm 1.1)^{* *}$ & $82.0( \pm 1.3)$ & $82.9( \pm 1.3)$ & $84.1( \pm 1.1)$ \\
\hline \multicolumn{6}{|l|}{ HepA } \\
\hline$\geq 1$ dose & $81.2( \pm 1.0)^{* *}$ & $81.5( \pm 1.1)$ & $83.1( \pm 1.2)^{* *}$ & $85.1( \pm 1.1)^{* *}$ & $85.8( \pm 1.1)$ \\
\hline$\geq 2$ doses & $52.2( \pm 1.4)^{* *}$ & $53.0( \pm 1.5)$ & $54.7( \pm 1.6)$ & $57.5( \pm 1.6)^{* *}$ & $59.6( \pm 1.5)$ \\
\hline Rotavirus $^{\dagger \dagger}$ & $67.3( \pm 1.3)^{* *}$ & $68.6( \pm 1.4)$ & $72.6( \pm 1.5)^{* * *}$ & $71.7( \pm 1.6)$ & $73.2( \pm 1.4)$ \\
\hline Combined series ${ }^{\S \S}$ & $68.5( \pm 1.3)^{* *}$ & $68.4( \pm 1.4)$ & $70.4( \pm 1.5)$ & $71.6( \pm 1.5)$ & $72.2( \pm 1.4)$ \\
\hline No vaccinations & $0.8( \pm 0.2)$ & $0.8( \pm 0.1)$ & $0.7( \pm 0.3)$ & $0.8( \pm 0.2)$ & $0.8( \pm 0.2)$ \\
\hline
\end{tabular}

Abbreviations: $\mathrm{Cl}=$ confidence interval; $\mathrm{DTaP}=$ diphtheria, tetanus toxoids, and acellular pertussis vaccine; $\mathrm{HepA}=$ hepatitis $\mathrm{A}$ vaccine; $\mathrm{HepB}=$ hepatitis $\mathrm{B}$ vaccine; $\mathrm{Hib}=$ Haemophilus influenzae type $\mathrm{b}$ vaccine; $\mathrm{MMR}=$ measles, mumps, and rubella vaccine; $\mathrm{PCV}=$ pneumococcal conjugate vaccine.

* For 2011, children born January 2008-May 2010; for 2012, children born January 2009-May 2011; for 2013, children born January 2010-May 2012; for 2014, children born January 2011-May 2013; and for 2015, children born January 2012-May 2014.

† Includes children who might have been vaccinated with diphtheria and tetanus toxoids vaccine, or diphtheria, tetanus toxoids, and pertussis vaccine.

$\S$ Hib Primary series: receipt of $\geq 2$ or $\geq 3$ doses, depending on product type received. Full series: receipt of $\geq 3$ or $\geq 4$ doses, depending on product type received (primary series and booster dose).

I One dose HepB administered between birth and age 3 days.

** Statistically significant $(p<0.05)$ change in coverage compared with previous year.

t+ Rotavirus vaccine includes $\geq 2$ or $\geq 3$ doses, depending on the product type received ( $\geq 2$ doses for Rotarix [RV1] and $\geq 3$ doses for RotaTeq [RV5]).

$\S \S$ The combined seven-vaccine series $\left(4: 3: 1: 3^{*}: 3: 1: 4\right)$ includes $\geq 4$ doses of DTaP, $\geq 3$ doses of poliovirus vaccine, $\geq 1$ dose of measles-containing vaccine, full series of Hib ( 3 or 4 doses, depending on product type), $\geq 3$ doses of hepatitis B vaccine, $\geq 1$ dose of varicella vaccine, and $\geq 4$ doses of PCV.

HepB (92.6\%), 3) $\geq 1$ dose of MMR (91.9\%), and 4) $\geq 1$ dose of varicella vaccine $(91.8 \%)$. Coverage remained below the target of $90 \%$ for $\geq 4$ doses of DTaP $(84.6 \%)$; the full series of Hib (82.7\%), and $\geq 4$ doses of PCV (84.1\%); below the $85 \%$ target for $\geq 2$ doses of hepatitis A vaccine (HepA) (59.6\%) and the HepB birth dose ${ }^{\mathbb{S}}(72.4 \%)$; and below the $80 \%$ target for rotavirus vaccination $(73.2 \%)$ and the combined seven-vaccine series $99(72.2 \%)$.

\footnotetext{
$\$ \$$ The Healthy People 2020 target for the birth dose (0-3 days) of HepB is $85 \%$, measured by annual birth cohort. For the three most recent completed birth cohorts examined by NIS, coverage with the birth dose of HepB was $71.8 \%$ for children born in 2010, $73.2 \%$ for children born in 2011 , and $73.3 \%$ for children born in 2012

99 The combined seven-vaccine series (4:3:1:3*:3:1:4) includes $\geq 4$ doses of DTaP/ diphtheria and tetanus toxoids vaccine/diphtheria, tetanus toxoids, and pertussis vaccine; $\geq 3$ doses of poliovirus vaccine; $\geq 1$ dose of measles-containing vaccine; $\geq 3$ or $\geq 4$ doses of $\mathrm{Hib}$ (depending upon product type of vaccine); $\geq 3$ doses of HepB; $\geq 1$ dose of varicella vaccine; and $\geq 4$ doses of PCV.
}

\section{Vaccination Coverage by Race/Ethnicity, Poverty Level, and MSA Status}

Compared with non-Hispanic white*** (white) children, non-Hispanic black (black) children had lower coverage with the full series of Hib, $\geq 4$ doses of PCV, and the rotavirus series (Table 2). Coverage differences between black and white children were not statistically significant after adjustment for poverty status (data not shown). Black, Hispanic, non-Hispanic Asian, and non-Hispanic American Indian/Alaska Native children had higher coverage with the HepB birth dose than did white children. Among Asian children, coverage with $\geq 4$ doses of DTaP, $\geq 3$ doses of poliovirus vaccine, $\geq 3$ doses of HepB, the HepB birth dose, and $\geq 2$ doses of HepA was higher than that for white children.

\footnotetext{
*** Child's race/ethnicity was reported by his/her parent or guardian. Children categorized in this report as white, black, American Indian/Alaska native, Asian, native Hawaiian/other Pacific Islander, or multiracial were identified as non-Hispanic by the parent or guardian. Children identified as multiracial had more than one race category identified. Persons identified as Hispanic might be of any race.
} 
TABLE 2. Estimated vaccination coverage among children aged $19-35$ months, by selected vaccines and doses, race/ethnicity, ${ }^{*}$ poverty level, ${ }^{\dagger}$ and Metropolitan Statistical Area (MSA) status ${ }^{\S}$ - National Immunization Survey, United States, $2015^{\natural}$

\begin{tabular}{|c|c|c|c|c|c|c|c|c|c|c|c|c|}
\hline \multirow[b]{3}{*}{ Vaccine/Dose } & \multicolumn{7}{|c|}{ Race/ethnicity } & \multicolumn{2}{|c|}{ Poverty level } & \multicolumn{3}{|c|}{ MSA status } \\
\hline & $\begin{array}{c}\text { White, } \\
\text { non-Hispanic } \\
\text { (Referent) }\end{array}$ & $\begin{array}{c}\text { Black, } \\
\text { non-Hispanic }\end{array}$ & Hispanic & $\begin{array}{c}\text { American } \\
\text { Indian/ } \\
\text { Alaska Native }\end{array}$ & Asian & $\begin{array}{c}\text { Native } \\
\text { Hawaiian or } \\
\text { other Pacific } \\
\text { Islander }\end{array}$ & Multiracial & $\begin{array}{c}\text { At or above } \\
\text { poverty } \\
\text { (Referent) }\end{array}$ & $\begin{array}{l}\text { Below } \\
\text { poverty }\end{array}$ & $\begin{array}{c}\text { MSA, } \\
\text { central city } \\
\text { (Referent) }\end{array}$ & $\begin{array}{c}\text { MSA, } \\
\text { non- } \\
\text { central city }\end{array}$ & Non-MSA \\
\hline & $\%(95 \% \mathrm{Cl})$ & $\%(95 \% \mathrm{Cl})$ & $\%(95 \% \mathrm{Cl})$ & $\%(95 \% \mathrm{Cl})$ & $\%(95 \% \mathrm{Cl})$ & $\%(95 \% \mathrm{Cl})$ & $\%(95 \% \mathrm{Cl})$ & $\%(95 \% \mathrm{Cl})$ & $\%(95 \% \mathrm{Cl})$ & $\%(95 \% \mathrm{Cl})$ & $\%(95 \% \mathrm{Cl})$ & $\%(95 \% \mathrm{Cl})$ \\
\hline \multicolumn{13}{|l|}{ DTaP** } \\
\hline$\geq 3$ doses & $94.8( \pm 0.8)$ & $94.3( \pm 1.6)$ & $95.5( \pm 1.2)$ & $92.3( \pm 4.7)$ & $97.3( \pm 1.6)^{\dagger \dagger}$ & $92.2( \pm 6.6)$ & $93.6( \pm 2.7)$ & $96.0( \pm 0.6)$ & $93.1( \pm 1.2)^{+\dagger}$ & $95.3( \pm 0.8)$ & $95.0( \pm 0.9)$ & $93.5( \pm 1.5)^{+\dagger}$ \\
\hline$\geq 4$ doses & $85.2( \pm 1.5)$ & $82.0( \pm 2.9)$ & $84.5( \pm 2.6)$ & $79.6( \pm 7.6)$ & $90.0( \pm 3.5)^{t \dagger}$ & —§§ & $82.5( \pm 3.8)$ & $87.1( \pm 1.3)$ & $80.2( \pm 2.2)^{\dagger \dagger}$ & $85.4( \pm 1.5)$ & $84.3( \pm 1.9)$ & $82.7( \pm 2.4)$ \\
\hline $\begin{array}{l}\text { Poliovirus } \\
\text { ( } \geq 3 \text { doses) }\end{array}$ & $93.1( \pm 0.9)$ & $93.3( \pm 1.7)$ & $94.5( \pm 1.3)$ & $91.8( \pm 4.7)$ & $96.9( \pm 1.7)^{\dagger \dagger}$ & $92.8( \pm 6.4)$ & $92.4( \pm 2.8)$ & $94.6( \pm 0.7)$ & $91.8( \pm 1.4)^{\dagger \dagger}$ & $93.9( \pm 1.0)$ & $94.0( \pm 1.0)$ & $91.7( \pm 1.8)^{\dagger \dagger}$ \\
\hline MMR ( $\geq 1$ dose) & $91.8( \pm 1.1)$ & $90.7( \pm 2.3)$ & $92.3( \pm 1.8)$ & $88.5( \pm 6.1)$ & $92.5( \pm 3.4)$ & $92.0( \pm 6.6)$ & $93.0( \pm 2.3)$ & $92.9( \pm 0.9)$ & $90.3( \pm 1.6)^{\dagger \dagger}$ & $92.4( \pm 1.2)$ & $91.7( \pm 1.3)$ & $90.7( \pm 1.8)$ \\
\hline \multicolumn{13}{|l|}{ Hibๆๆ } \\
\hline$\geq 3$ doses & $93.3( \pm 0.9)$ & $92.1( \pm 2.0)$ & $94.0( \pm 1.4)$ & $88.2( \pm 5.7)$ & $93.7( \pm 2.7)$ & $91.8( \pm 6.8)$ & $92.0( \pm 2.8)$ & $94.7( \pm 0.7)$ & $90.5( \pm 1.4)^{\dagger \dagger}$ & $93.6( \pm 1.0)$ & $93.3( \pm 1.1)$ & $91.8( \pm 1.6)$ \\
\hline Primary series & $94.4( \pm 0.8)$ & $93.3( \pm 1.8)$ & $95.1( \pm 1.3)$ & $89.8( \pm 5.6)$ & $94.8( \pm 2.2)$ & $92.8( \pm 6.4)$ & $93.1( \pm 2.7)$ & $95.6( \pm 0.7)$ & $91.9( \pm 1.3)^{+\dagger}$ & $94.6( \pm 0.9)$ & $94.3( \pm 1.0)$ & $93.2( \pm 1.5)$ \\
\hline Full series & $83.0( \pm 1.5)$ & $78.9( \pm 3.1)^{\dagger \dagger}$ & $83.0( \pm 2.6)$ & $81.4( \pm 7.3)$ & $87.0( \pm 3.9)$ & —§§ & $82.4( \pm 3.7)$ & $85.5( \pm 1.3)$ & $78.1( \pm 2.2)^{\dagger \dagger}$ & $82.3( \pm 1.6)$ & $83.6( \pm 1.8)$ & $80.9( \pm 2.4)$ \\
\hline \multicolumn{13}{|l|}{ НерB } \\
\hline$\geq 3$ doses & $92.0( \pm 1.1)$ & $93.3( \pm 1.8)$ & $93.2( \pm 1.5)$ & $92.4( \pm 4.9)$ & $95.5( \pm 2.3)^{t \dagger}$ & $94.1( \pm 5.5)$ & $91.4( \pm 3.6)$ & $92.7( \pm 1.0)$ & $92.5( \pm 1.3)$ & $92.9( \pm 1.1)$ & $92.5( \pm 1.2)$ & $92.1( \pm 1.6)$ \\
\hline Birth dose $\mathrm{e}^{* * *}$ & $68.2( \pm 1.8)$ & $74.2( \pm 3.6)^{\dagger \dagger}$ & $77.8( \pm 3.2)^{\dagger \dagger}$ & $80.7( \pm 8.4)^{\dagger \dagger}$ & $76.7( \pm 5.5)^{\dagger \dagger}$ & —§§ & $72.8( \pm 4.9)$ & $70.2( \pm 1.6)$ & $76.3( \pm 2.5)^{\dagger+}$ & $72.1( \pm 2.1)$ & $71.7 \pm 2.2)$ & $75.6( \pm 2.8)^{\dagger \dagger}$ \\
\hline $\begin{array}{l}\text { Varicella } \\
\qquad(\geq 1 \text { dose) }\end{array}$ & $91.2( \pm 1.1)$ & $91.8( \pm 2.1)$ & $92.7( \pm 1.8)$ & $87.8( \pm 6.0)$ & $93.4( \pm 2.9)$ & $91.8( \pm 6.8)$ & $92.1( \pm 2.5)$ & $92.5( \pm 0.9)$ & $90.6( \pm 1.6)$ & $92.5( \pm 1.2)$ & $91.5( \pm 1.2)$ & $89.9( \pm 1.9)^{\dagger \dagger}$ \\
\hline \multicolumn{13}{|l|}{ PCV } \\
\hline$\geq 3$ doses & $93.2( \pm 1.0)$ & $92.5( \pm 1.9)$ & $94.4( \pm 1.6)$ & $89.7( \pm 5.4)$ & $92.4( \pm 2.9)$ & $90.6( \pm 7.5)$ & $92.5( \pm 2.8)$ & $94.6( \pm 0.8)$ & $91.2( \pm 1.4)^{+\dagger}$ & $93.1( \pm 1.1)$ & $93.9( \pm 1.0)$ & $91.8( \pm 1.7)$ \\
\hline$\geq 4$ doses & $85.0( \pm 1.5)$ & $81.4( \pm 2.9)^{\dagger \dagger}$ & $84.0( \pm 2.5)$ & $77.1( \pm 7.9)$ & $85.0( \pm 4.1)$ & —§§ & $83.7( \pm 3.6)$ & $87.2( \pm 1.2)$ & $78.9( \pm 2.2)^{\dagger \dagger}$ & $83.9( \pm 1.6)$ & $85.5( \pm 1.7)$ & $80.4( \pm 2.5)^{\dagger \dagger}$ \\
\hline $\begin{array}{l}\text { HepA } \\
\text { ( } \geq 2 \text { doses) }\end{array}$ & $58.7( \pm 1.9)$ & $59.3( \pm 3.9)$ & $60.9( \pm 3.5)$ & $61.3( \pm 9.5)$ & $67.8( \pm 6.2)^{\dagger \dagger}$ & —§§ & $54.1( \pm 5.3)$ & $61.7( \pm 1.7)$ & $56.0( \pm 2.8)^{\dagger \dagger}$ & $60.5( \pm 2.2)$ & $59.6( \pm 2.4)$ & $55.7( \pm 3.2)^{\dagger \dagger}$ \\
\hline Rotavirus ${ }^{+\dagger \dagger}$ & $74.6( \pm 1.7)$ & $69.7( \pm 3.6)^{\dagger+}$ & $72.9( \pm 3.2)$ & —§§ & $75.6( \pm 5.4)$ & —§§ & $70.6( \pm 5.2)$ & $76.8( \pm 1.6)$ & $66.8( \pm 2.7)^{+\dagger}$ & $72.7( \pm 2.1)$ & $75.1( \pm 2.1)$ & $68.6( \pm 3.0)^{t+}$ \\
\hline $\begin{array}{c}\text { Combined } \\
\text { series }^{\S \S \S}\end{array}$ & $72.7( \pm 1.8)$ & $69.1( \pm 3.6)$ & $71.7( \pm 3.2)$ & $68.2( \pm 9.0)$ & $77.9( \pm 4.9)$ & —§§ & $73.7( \pm 4.6)$ & $74.7( \pm 1.6)$ & $68.7( \pm 2.5)^{\dagger \dagger}$ & $72.5( \pm 2.0)$ & $72.5( \pm 2.3)$ & $70.2( \pm 2.9)$ \\
\hline
\end{tabular}

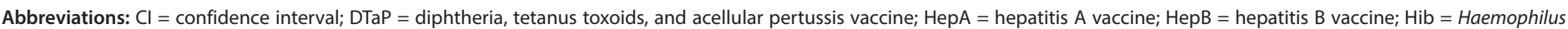
influenzae type $b$ vaccine; MMR = measles, mumps, and rubella vaccine; PCV = pneumococcal conjugate vaccine.

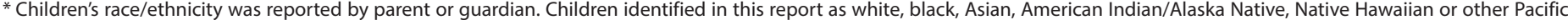

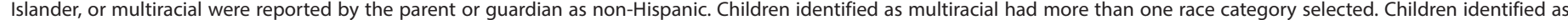
Hispanic might be of any race.



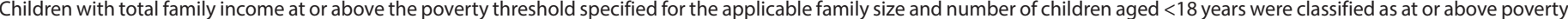

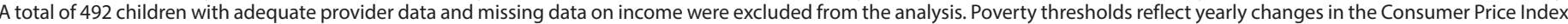
(http://www.census.gov/hhes/www/poverty.html).

$\S$ Metropolitan Statistical Area as defined by the US Office of Management and Budget (https://www.whitehouse.gov/sites/default/files/omb/bulletins/2015/15-01.pdf).

I Children in the 2015 National Immunization Survey were born January 2012-May 2014

** Includes children who might have been vaccinated with diphtheria and tetanus toxoids vaccine, or diphtheria, tetanus toxoids, and pertussis vaccine.

t+ Statistically significant $(p<0.05)$ difference from referent group.

$\S \S$ Estimate not available because the unweighted sample size for the denominator was $<30$, or $95 \% \mathrm{Cl}$ half-width/estimate $>0.588$, or $95 \% \mathrm{Cl}$ half-width was $\geq 10$.

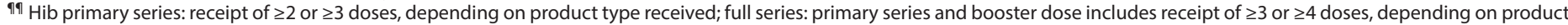
type received.

*** One dose HepB administered from birth through age 3 days.

${ }^{\dagger+\dagger}$ Includes $\geq 2$ or $\geq 3$ doses, depending on product type received ( $\geq 2$ doses for Rotarix [RV1], or $\geq 3$ doses for RotaTeq [RV5]).

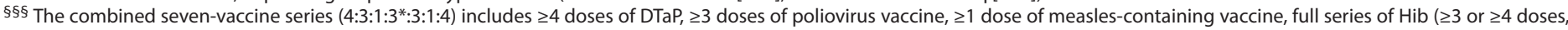
depending on type), $\geq 3$ doses of HepB, $\geq 1$ dose of varicella vaccine, and $\geq 4$ doses of PCV.

Children living below the federal poverty level had lower coverage with nearly all vaccines compared with children living at or above the poverty level (Table 2). As in 2014, coverage with $\geq 4$ doses of DTaP, $\geq 3$ doses of poliovirus vaccine, $\geq 1$ dose of MMR, the primary and full series of Hib, $\geq 4$ doses of PCV, $\geq 2$ doses of HepA, the rotavirus series and the combined sevenvaccine series was lower among children below the poverty level. The difference in coverage levels ranged from 2.6 to 10.0 percentage points; for five vaccines/doses ( $\geq 4$ doses of DTaP, the full series of Hib, $\geq 4$ doses of PCV, $\geq 2$ doses of HepA, the rotavirus series) and the combined seven-vaccine series, the disparity exceeded 5.0 percentage points.
Coverage differed by MSA status for several vaccines in 2015 (Table 2). Children living in a non-MSA had lower coverage with $\geq 3$ doses of $\mathrm{DTaP}, \geq 3$ doses of poliovirus vaccine, $\geq 1$ dose of varicella vaccine, $\geq 4$ doses of $\mathrm{PCV}$, $\geq 2$ doses of HepA, and rotavirus vaccine than did children in an MSA central city. Logistic regression analyses did not identify any statistically significant interaction between poverty status and MSA status for any of the vaccines monitored by NIS. In all cases, poverty status remained independently associated with vaccination coverage after adjustment for MSA status. 
TABLE 3. Estimated vaccination coverage with selected individual vaccines and a combined vaccine series* among children aged $19-35$ months, overall and by U.S. Department of Health and Human Services (HHS) region and state and local area — National Immunization Survey, United States, $2015^{\dagger}$

\begin{tabular}{|c|c|c|c|c|c|c|}
\hline \multirow{3}{*}{$\begin{array}{l}\text { National, HHS region, state, and } \\
\text { local area }\end{array}$} & \multicolumn{6}{|c|}{ Vaccine/Vaccine series } \\
\hline & MMR ( $\geq 1$ dose) & $\mathrm{DTaP}(\geq 4 \text { doses })^{\S}$ & Hep B (birth dose) & HepA ( $\geq 2$ doses) & Rotavirus** & $\begin{array}{c}\text { Combined } \\
\text { vaccine series }\end{array}$ \\
\hline & $\%(95 \% \mathrm{Cl})$ & $\%(95 \% \mathrm{Cl})$ & $\%(95 \% \mathrm{Cl})$ & $\%(95 \% \mathrm{Cl})$ & $\%(95 \% \mathrm{Cl})$ & $\%(95 \% \mathrm{Cl})$ \\
\hline U.S. overall & $91.9( \pm 0.8)$ & $84.6( \pm 1.1)$ & $72.4( \pm 1.4)$ & $59.6( \pm 1.5)$ & $73.2( \pm 1.4)$ & $72.2( \pm 1.4)$ \\
\hline HHS Region I & $94.1( \pm 2.1)$ & $88.9( \pm 2.7)$ & $76.3( \pm 3.3)$ & $65.4( \pm 3.9)$ & $80.7( \pm 3.2)$ & $77.8( \pm 3.3)$ \\
\hline Connecticut & $97.5( \pm 2.4)$ & $90.8( \pm 4.5)$ & $81.8( \pm 6.2)$ & $72.0( \pm 7.3)$ & $77.9( \pm 6.7)$ & $80.6( \pm 6.0)$ \\
\hline Maine & $96.0( \pm 3.1)$ & $92.0( \pm 5.0)$ & $68.7( \pm 7.7)$ & $53.8( \pm 8.3)$ & $71.1( \pm 7.7)$ & $71.8( \pm 7.9)^{\dagger \dagger}$ \\
\hline Massachusetts & $91.8( \pm 4.0)$ & $87.2( \pm 5.1)$ & $78.4( \pm 5.8)$ & $65.7( \pm 6.9)$ & $83.5( \pm 5.4)$ & $78.5( \pm 6.0)$ \\
\hline New Hampshire & $93.4( \pm 3.9)$ & $88.4( \pm 5.4)$ & $72.0( \pm 7.0)$ & $60.2( \pm 7.7)$ & $80.9( \pm 6.2)$ & $74.1( \pm 7.1)$ \\
\hline Rhode Island & $94.5( \pm 3.2)$ & $90.5( \pm 4.1)$ & $73.2( \pm 6.4)$ & $65.1( \pm 6.9)$ & $87.6( \pm 4.9)$ & $77.2( \pm 6.0)$ \\
\hline Vermont & $95.5( \pm 2.7)$ & $89.2( \pm 4.2)$ & $49.4( \pm 6.7)$ & $57.1( \pm 6.7)$ & $72.7( \pm 6.2)$ & $75.6( \pm 5.9)$ \\
\hline HHS Region II & $92.6( \pm 2.2)$ & $88.1( \pm 2.7)$ & $60.6( \pm 4.0)$ & $53.4( \pm 4.1)$ & $73.7( \pm 3.8)^{\S \S}$ & $73.4( \pm 3.7)$ \\
\hline New Jersey & $92.8( \pm 4.4)$ & $89.8( \pm 4.8)$ & $63.9( \pm 7.2)$ & $58.3( \pm 7.4)$ & $75.2( \pm 6.8)$ & $76.5( \pm 6.5)$ \\
\hline New York & $92.5( \pm 2.6)$ & $87.4( \pm 3.3)$ & $59.0( \pm 4.7)$ & $51.2( \pm 5.0)$ & $73.0( \pm 4.5)$ & $71.9( \pm 4.4)$ \\
\hline City of New York & $94.1( \pm 2.9)$ & $85.5( \pm 5.0)$ & $53.4( \pm 6.8)$ & $47.8( \pm 6.9)$ & $71.1( \pm 6.4)$ & $68.2( \pm 6.5)$ \\
\hline Rest of state (NY) & $90.9( \pm 4.3)$ & $89.2( \pm 4.3)$ & $64.6( \pm 6.6)$ & $54.6( \pm 7.2)$ & $75.0( \pm 6.4)$ & $75.7( \pm 6.1)$ \\
\hline HHS Region III & $89.6( \pm 2.5)$ & $85.5( \pm 2.7)$ & $72.5( \pm 3.6)^{\dagger+}$ & $61.5( \pm 3.8)$ & $72.7( \pm 3.6)$ & $71.0( \pm 3.6)$ \\
\hline Delaware & $97.2( \pm 2.6)^{\S \S}$ & $89.9( \pm 4.5)$ & $76.0( \pm 6.7)$ & $67.6( \pm 7.3)$ & $81.5( \pm 6.1)$ & $79.3( \pm 6.1)$ \\
\hline District of Columbia & $92.4( \pm 3.8)$ & $86.1( \pm 5.0)$ & $72.7( \pm 5.8)$ & $67.9( \pm 6.5)$ & $73.0( \pm 6.2)$ & $76.3( \pm 6.0)$ \\
\hline Maryland & $95.4( \pm 2.5)$ & $87.6( \pm 4.5)$ & $79.0( \pm 6.6)$ & $63.0( \pm 7.2)$ & $76.8( \pm 6.4)$ & $76.8( \pm 5.9)$ \\
\hline Pennsylvania & $90.9( \pm 3.7)$ & $88.7( \pm 3.8)$ & $73.2( \pm 6.0)$ & $64.6( \pm 6.3)$ & $74.3( \pm 5.9)$ & $72.8( \pm 5.8)$ \\
\hline Philadelphia & $93.2( \pm 3.5)$ & $87.2( \pm 4.5)$ & $77.3( \pm 6.1)$ & $65.4( \pm 6.9)$ & $71.5( \pm 6.4)$ & $76.1( \pm 6.0)$ \\
\hline Rest of state (PA) & $90.5( \pm 4.4)$ & $88.9( \pm 4.4)$ & $72.5( \pm 7.0)$ & $64.4( \pm 7.4)$ & $74.9( \pm 6.9)$ & $72.2( \pm 6.8)$ \\
\hline Virginia & $83.4( \pm 6.7)$ & $80.6( \pm 7.0)$ & $67.3( \pm 8.1)$ & $54.1( \pm 8.3)$ & $67.1( \pm 8.3)$ & $64.4( \pm 8.3)$ \\
\hline West Virginia & $86.7( \pm 5.7)$ & $78.6( \pm 6.8)$ & $68.3( \pm 7.4)$ & $65.7( \pm 7.7)^{\S \S}$ & $69.6( \pm 7.5)$ & $64.9( \pm 7.8)$ \\
\hline HHS Region IV & $91.3( \pm 1.9)$ & $83.3( \pm 2.3)$ & $70.9( \pm 2.9)$ & $55.8( \pm 3.1)$ & $69.8( \pm 3.0)$ & $71.2( \pm 2.9)$ \\
\hline Alabama & $95.2( \pm 3.5)$ & $82.2( \pm 6.4)$ & $83.2( \pm 5.4)$ & $57.6( \pm 7.6)$ & $76.2( \pm 6.9)$ & $70.6( \pm 7.1)$ \\
\hline Florida & $90.4( \pm 5.0)$ & $86.0( \pm 5.2)$ & $56.9( \pm 7.6)$ & $54.6( \pm 7.5)$ & $63.8( \pm 7.5)$ & $66.6( \pm 7.2)$ \\
\hline Georgia & $90.5( \pm 4.7)$ & $82.3( \pm 6.0)$ & $80.5( \pm 5.7)$ & $62.0( \pm 7.6)$ & $73.8( \pm 7.0)$ & $75.6( \pm 6.7)$ \\
\hline Kentucky & $91.6( \pm 4.1)$ & $87.0( \pm 4.8)$ & $75.3( \pm 6.7)$ & $48.3( \pm 7.3)$ & $65.3( \pm 7.1)$ & $73.0( \pm 6.5)$ \\
\hline Mississippi & $89.8( \pm 5.3)$ & $79.6( \pm 7.0)$ & $77.0( \pm 7.3)$ & $41.2( \pm 8.1)$ & $65.9( \pm 8.0)$ & $70.6( \pm 7.5)$ \\
\hline North Carolina & $94.3( \pm 3.3)$ & $83.9( \pm 5.8)$ & $81.3( \pm 6.0)$ & $56.2( \pm 7.5)$ & $75.9( \pm 6.7)^{\dagger \dagger}$ & $76.4( \pm 6.5)$ \\
\hline South Carolina & $88.5( \pm 5.1)$ & $77.5( \pm 7.0)$ & $68.9( \pm 7.0)$ & $54.5( \pm 7.6)$ & $69.8( \pm 7.1)$ & $68.2( \pm 7.3)$ \\
\hline Tennessee & $90.2( \pm 4.0)^{+\dagger}$ & $81.2( \pm 6.5)$ & $64.7( \pm 7.9)^{\dagger \dagger}$ & $59.5( \pm 8.0)$ & $70.9( \pm 7.6)$ & $70.1( \pm 7.5)$ \\
\hline HHS Region V & $90.9( \pm 1.8)$ & $84.2( \pm 2.3)$ & $75.2( \pm 2.5)$ & $59.9( \pm 3.0)$ & $73.2( \pm 2.8)$ & $70.2( \pm 2.8)$ \\
\hline Illinois & $91.6( \pm 2.8)$ & $85.0( \pm 3.8)$ & $71.2( \pm 4.6)$ & $57.9( \pm 4.9)$ & $75.4( \pm 4.5)$ & $70.8( \pm 4.7)$ \\
\hline City of Chicago & $90.5( \pm 4.7)$ & $86.2( \pm 5.9)$ & $82.9( \pm 5.9)$ & $62.7( \pm 8.0)$ & $78.3( \pm 6.8)^{\S \S}$ & $72.8( \pm 7.3)$ \\
\hline Rest of state (IL) & $91.9( \pm 3.4)$ & $84.6( \pm 4.6)$ & $67.2( \pm 5.8)$ & $56.3( \pm 6.0)$ & $74.4( \pm 5.5)$ & $70.1( \pm 5.8)$ \\
\hline Indiana & $92.0( \pm 4.4)$ & $85.3( \pm 5.7)$ & $80.0( \pm 6.3)$ & $65.3( \pm 7.4)^{\S \S}$ & $72.6( \pm 7.2)$ & $74.7( \pm 7.0)$ \\
\hline Michigan & $90.6( \pm 4.5)$ & $84.9( \pm 5.5)$ & $80.0( \pm 5.9)$ & $64.1( \pm 7.2)^{\S \S}$ & $65.5( \pm 7.7)$ & $67.6( \pm 7.3)$ \\
\hline Minnesota & $92.6( \pm 3.6)$ & $85.4( \pm 5.0)$ & $67.8( \pm 6.5)$ & $65.4( \pm 6.6)$ & $82.6( \pm 5.4)$ & $73.2( \pm 6.4)$ \\
\hline Ohio & $88.1( \pm 5.6)^{\dagger \dagger}$ & $80.9( \pm 6.8)$ & $77.9( \pm 6.5)$ & $53.1( \pm 8.2)$ & $71.8( \pm 7.6)$ & $68.3( \pm 7.9)$ \\
\hline Wisconsin & $92.4( \pm 4.3)$ & $85.2( \pm 5.7)$ & $73.1( \pm 6.6)$ & $58.6( \pm 7.5)$ & $75.3( \pm 6.7)$ & $68.8( \pm 7.1)$ \\
\hline HHS Region VI & $92.3( \pm 1.8)$ & $82.4( \pm 2.7)$ & $76.5( \pm 2.9)$ & $63.9( \pm 3.2)^{\S \S}$ & $73.5( \pm 2.9)$ & $71.2( \pm 3.1)$ \\
\hline Arkansas & $90.2( \pm 5.0)$ & $76.4( \pm 6.9)$ & $80.6( \pm 6.2)$ & $54.2( \pm 8.0)$ & $68.2( \pm 7.4)$ & $66.6( \pm 7.5)$ \\
\hline Louisiana & $92.6( \pm 4.3)$ & $84.4( \pm 5.7)$ & $75.3( \pm 7.1)$ & $59.0( \pm 8.0)$ & $67.7( \pm 7.6)$ & $70.8( \pm 7.6)$ \\
\hline New Mexico & $89.7( \pm 5.3)$ & $84.8( \pm 6.0)$ & $67.8( \pm 7.5)$ & $62.7( \pm 7.9)$ & $73.8( \pm 6.9)$ & $70.1( \pm 7.9)$ \\
\hline Oklahoma & $92.6( \pm 5.1)$ & $85.7( \pm 6.3)$ & $80.4( \pm 5.9)$ & $71.6( \pm 7.4)^{\S \S}$ & $67.2( \pm 8.3)$ & $75.4( \pm 7.3)$ \\
\hline Texas & $92.5( \pm 2.4)$ & $82.1( \pm 3.6)$ & $76.4( \pm 3.9)$ & $64.6( \pm 4.3)$ & $75.7( \pm 3.9)^{\S \S}$ & $71.2( \pm 4.2)^{\S \S}$ \\
\hline Bexar County & $89.2( \pm 5.0)$ & $80.1( \pm 6.1)$ & $72.5( \pm 6.7)$ & $64.2( \pm 7.3)$ & $70.7( \pm 7.0)$ & $67.5( \pm 7.1)$ \\
\hline City of Houston & $92.8( \pm 3.9)$ & $80.5( \pm 6.5)$ & $82.6( \pm 5.7)$ & $64.5( \pm 7.6)$ & $74.8( \pm 6.8)$ & $70.5( \pm 7.3)$ \\
\hline El Paso County & $90.6( \pm 4.5)$ & $82.8( \pm 6.1)$ & $76.5( \pm 7.0)$ & $73.5( \pm 6.7)^{\S \S}$ & $73.4( \pm 7.1)$ & $71.6( \pm 7.1)$ \\
\hline Hidalgo County & $86.9( \pm 5.2)$ & $82.4( \pm 5.8)$ & $89.5( \pm 4.8)$ & $64.1( \pm 6.7)$ & $73.1( \pm 6.4)$ & $71.6( \pm 6.5)$ \\
\hline Rest of state $(\mathrm{TX})^{\text {ๆq }}$ & $93.3( \pm 3.1)$ & $82.5( \pm 4.8)$ & $74.9( \pm 5.2)$ & $64.2( \pm 5.7)$ & $76.6( \pm 5.1)$ & $71.6( \pm 5.6)$ \\
\hline HHS Region VII & $93.2( \pm 2.1)$ & $85.5( \pm 3.0)$ & $77.2( \pm 3.6)$ & $63.5( \pm 4.0)^{\S \S}$ & $75.7( \pm 3.6)$ & $73.8( \pm 3.7)$ \\
\hline lowa & $95.5( \pm 2.4)$ & $88.9( \pm 4.0)$ & $78.2( \pm 5.3)$ & $64.7( \pm 6.5)$ & $75.1( \pm 5.9)$ & $77.9( \pm 5.5)$ \\
\hline Kansas & $92.3( \pm 4.1)$ & $86.8( \pm 4.7)$ & $83.3( \pm 5.4)$ & $67.9( \pm 6.8)$ & $77.0( \pm 6.3)$ & $75.2( \pm 6.3)$ \\
\hline Missouri & $91.6( \pm 4.3)$ & $82.6( \pm 6.3)$ & $75.0( \pm 7.4)$ & $57.2( \pm 7.9)^{\S \S}$ & $76.1( \pm 7.0)$ & $71.0( \pm 7.4)$ \\
\hline Nebraska & $95.6( \pm 3.0)$ & $86.9( \pm 5.0)$ & $72.5( \pm 6.8)$ & $72.8( \pm 6.5)$ & $73.0( \pm 6.4)$ & $73.8( \pm 6.3)$ \\
\hline
\end{tabular}

See table footnotes on the next page. 
TABLE 3. (Continued) Estimated vaccination coverage with selected individual vaccines and a combined vaccine series* among children aged 19-35 months, overall and by U.S. Department of Health and Human Services (HHS) region and state and local area — National Immunization Survey, United States, $2015^{\dagger}$

\begin{tabular}{|c|c|c|c|c|c|c|}
\hline \multirow{3}{*}{$\begin{array}{l}\text { National, HHS region, state, and } \\
\text { local area }\end{array}$} & \multicolumn{6}{|c|}{ Vaccine/Vaccine series } \\
\hline & MMR ( $\geq 1$ dose) & DTaP $(\geq 4 \text { doses })^{\S}$ & Hep B (birth dose) & HepA ( $\geq 2$ doses) & Rotavirus** & $\begin{array}{c}\text { Combined } \\
\text { vaccine series }\end{array}$ \\
\hline & $\%(95 \% \mathrm{Cl})$ & $\%(95 \% \mathrm{Cl})$ & $\%(95 \% \mathrm{Cl})$ & $\%(95 \% \mathrm{Cl})$ & $\%(95 \% \mathrm{Cl})$ & $\%(95 \% \mathrm{Cl})$ \\
\hline HHS Region VIII & $91.8( \pm 2.3)$ & $83.8( \pm 2.9)$ & $75.8( \pm 3.4)$ & $57.8( \pm 4.0)$ & $74.9( \pm 3.6)$ & $72.7( \pm 3.6)$ \\
\hline Colorado & $93.6( \pm 3.6)$ & $86.1( \pm 5.0)$ & $73.1( \pm 6.4)$ & $59.7( \pm 7.3)$ & $75.9( \pm 6.5)$ & $75.4( \pm 6.2)$ \\
\hline Montana & $91.7( \pm 3.7)$ & $78.6( \pm 6.5)$ & $73.8( \pm 6.5)$ & $43.9( \pm 7.3)$ & $67.3( \pm 7.0)$ & $68.1( \pm 7.1)$ \\
\hline North Dakota & $92.8( \pm 3.8)$ & $85.7( \pm 5.1)$ & $87.5( \pm 4.7)$ & $66.3( \pm 6.9)$ & $79.8( \pm 6.2)$ & $80.2( \pm 5.7)$ \\
\hline South Dakota & $91.4( \pm 4.8)$ & $83.1( \pm 6.0)$ & $71.4( \pm 7.3)$ & $53.0( \pm 7.8)$ & $73.4( \pm 7.0)$ & $75.6( \pm 6.8)$ \\
\hline Utah & $89.2( \pm 5.2)$ & $82.0( \pm 5.8)$ & $79.6( \pm 6.0)$ & $59.8( \pm 7.6)$ & $74.5( \pm 6.7)$ & $68.1( \pm 7.3)$ \\
\hline Wyoming & $93.5( \pm 3.5)$ & $83.2( \pm 6.1)$ & $67.2( \pm 7.3)$ & $45.8( \pm 7.8)^{\S \S}$ & $76.4( \pm 6.6)$ & $73.3( \pm 7.1)$ \\
\hline HHS Region IX & $92.6( \pm 3.1)$ & $84.9( \pm 4.6)$ & $70.9( \pm 5.8)$ & $60.3( \pm 6.2)$ & $74.1( \pm 5.6)$ & $74.4( \pm 5.5)$ \\
\hline Arizona & $90.6( \pm 4.1)$ & $83.7( \pm 5.8)$ & $78.0( \pm 6.0)$ & $58.6( \pm 7.4)$ & $75.1 \pm 6.6)$ & $72.3( \pm 6.7)$ \\
\hline California & $92.8( \pm 3.9)$ & $85.2( \pm 5.7)$ & $69.1( \pm 7.4)$ & $60.8( \pm 7.8)$ & $74.6( \pm 7.1)$ & $75.0( \pm 6.9)$ \\
\hline Hawaii & $94.7( \pm 3.3)$ & $83.2( \pm 5.4)$ & $75.5( \pm 6.3)$ & $51.4( \pm 7.0)$ & $72.5( \pm 6.4)$ & $73.8( \pm 6.3)$ \\
\hline Nevada & $93.7( \pm 3.7)$ & $84.7( \pm 5.7)$ & $77.7( \pm 6.8)$ & $61.9( \pm 7.5)$ & $65.5( \pm 7.6)$ & $71.3( \pm 7.0)$ \\
\hline HHS Region X & $94.1( \pm 1.8)^{\S \S}$ & $85.6( \pm 3.1)$ & $71.0( \pm 3.9)$ & $58.8( \pm 4.4)$ & $73.4( \pm 4.0)$ & $73.0( \pm 3.9)$ \\
\hline Alaska & $89.7( \pm 4.0)$ & $79.7( \pm 5.2)$ & $65.7( \pm 6.1)^{\S \S}$ & $56.5( \pm 6.5)$ & $72.0( \pm 5.9)$ & $66.3( \pm 6.2)$ \\
\hline Idaho & $91.2( \pm 4.0)$ & $81.0( \pm 5.9)$ & $69.8( \pm 6.9)$ & $58.8( \pm 7.3)$ & $74.5( \pm 6.6)$ & $71.6( \pm 6.8)$ \\
\hline Oregon & $94.1( \pm 3.7)^{\S \S}$ & $85.8( \pm 5.7)$ & $72.5( \pm 7.0)^{\S \S}$ & $61.8( \pm 8.0)$ & $72.9( \pm 7.3)$ & $67.4( \pm 7.8)$ \\
\hline Washington & $95.3( \pm 2.6)^{\S \S}$ & $87.3( \pm 4.7)$ & $71.2( \pm 6.2)$ & $57.5( \pm 7.0)$ & $73.6( \pm 6.3)$ & $77.1( \pm 5.7)$ \\
\hline Range & $(83.4-97.5)$ & (76.4-92.0) & (49.4-87.5) & $(41.2-72.8)$ & $(63.8-87.6)$ & $(64.4-80.6)$ \\
\hline \multicolumn{7}{|l|}{ Territory } \\
\hline Guam*** & $91.7( \pm 3.6)$ & $73.3( \pm 6.1)$ & $82.3( \pm 5.0)$ & $49.4( \pm 6.9)$ & $40.6( \pm 6.5)$ & $52.9( \pm 7.0)$ \\
\hline Puerto Rico*** & $91.4( \pm 3.9)$ & $82.0( \pm 5.8)$ & $72.7( \pm 7.6)$ & $50.7( \pm 8.1)$ & $64.9( \pm 7.6)$ & $61.0( \pm 8.1)$ \\
\hline U.S. Virgin Islands ${ }^{* * *}$ & $77.2( \pm 5.5)$ & $65.8( \pm 6.3)$ & $79.4( \pm 5.3)$ & $36.8( \pm 6.5)$ & $43.3( \pm 6.9)$ & $50.7( \pm 6.7)$ \\
\hline
\end{tabular}

Abbreviations: $\mathrm{Cl}=$ confidence interval; $\mathrm{DTaP}=$ diphtheria, tetanus toxoids and acellular pertussis vaccine; HepA = hepatitis $\mathrm{A}$ vaccine; HepB = hepatitis $\mathrm{B}$ vaccine; $\mathrm{Hib}=$ Haemophilus influenzae type $\mathrm{b}$ vaccine; $\mathrm{MMR}=$ measles, mumps, and rubella vaccine; $\mathrm{PCV}=$ pneumococcal conjugate vaccine.

* The combined seven-vaccine series ( $\left.4: 3: 1: 3^{*}: 3: 1: 4\right)$ includes $\geq 4$ doses of DTaP, $\geq 3$ doses of poliovirus vaccine, $\geq 1$ doses of measles-containing vaccine, full series of Hib vaccine ( $\geq 3$ or $\geq 4$ doses, depending on product type), $\geq 3$ doses of HepB, $\geq 1$ dose of varicella vaccine, and $\geq 4$ doses of PCV.

† Children in the 2015 National Immunization Survey were born January 2012-May 2014.

$\S$ Includes children who might have been vaccinated with diphtheria and tetanus toxoids vaccine, or diphtheria, tetanus toxoids and pertussis vaccine

I One dose HepB administered from birth through age 3 days.

** Either $\geq 2$ or $\geq 3$ doses of rotavirus vaccine, depending on product type received ( $\geq 2$ doses for Rotarix [RV1] or $\geq 3$ doses for RotaTeq [RV5]).

t+ Statistically significant decrease in coverage compared to $2014(p<0.05)$.

$\S$ Statistically significant increase in coverage compared to $2014(p<0.05)$.

११ No comparison was made to coverage in 2014; Hidalgo County was not sampled in 2014 and "rest of state" is not comparable between the two years.

*** Children from Guam ( $n=467)$, Puerto Rico $(n=617)$, and the U.S. Virgin Islands $(n=580)$ were excluded from the national estimates. Guam and U.S. Virgin Islands were not sampled in 2014.

\section{Vaccination Coverage by Geographic Area}

Vaccination coverage in 2015 varied considerably by geographic area (Table 3), differing across states by as much as 38.1 percentage points. Coverage with $\geq 1$ dose of MMR ranged from $83.4 \%$ (Virginia) to $97.5 \%$ (Connecticut). The lowest estimated coverage with $\geq 4$ doses of DTaP was $76.4 \%$ (Arkansas) and the highest was $92.0 \%$ (Maine). The largest discrepancy among states was for the HepB birth dose, with a low of $49.4 \%$ (Vermont) and a high of $87.5 \%$ (North Dakota). Coverage with $\geq 2$ doses of HepA varied widely, ranging from $41.2 \%$ (Mississippi) to $72.8 \%$ (Nebraska). The lowest state-specific estimate for rotavirus series coverage was $63.8 \%$ (Florida) and the highest $87.6 \%$ (Rhode Island). Coverage with the combined seven-vaccine series ranged from $64.4 \%$ (Virginia) to $80.6 \%$ (Connecticut).

\section{Discussion}

Nationally, coverage with vaccines recommended by the Advisory Committee on Immunization Practices (ACIP) for children aged 19-35 months remains high and stable. The burden of most vaccine-preventable diseases is low in this population. Furthermore, Healthy People 2020 coverage targets continue to be met for poliovirus vaccine, MMR, HepB, and varicella vaccine. For other vaccines, coverage levels remained stable, and increased activities are needed to reach target coverage levels. Nationally, large disparities in coverage exist for children living below the poverty level compared with children living at or above the poverty level, with differences of 7-10 percentage points for $\geq 4$ doses of DTaP, Hib (full series), $\geq 4$ doses of PCV, and rotavirus vaccination. Disparities between racial/ethnic groups were observed, but 
these diminished in magnitude when poverty status and MSA were accounted for in the analysis.

The widespread, persistent and often sizeable vaccination coverage disparities between children living below poverty and children living at or above the poverty level have been observed for many vaccines monitored by NIS since at least 2009 and are concerning. Based on 2015 data, an estimated $32.9 \%(95 \% \mathrm{CI}=31.4 \%-34.4 \%)$ of U.S. children aged 19-35 months were living below the poverty level. The Vaccines for Children (VFC) program ${ }^{\dagger \dagger}$ has provided free vaccine to many uninsured, Medicaid-eligible and other children aged $\leq 18$ years who would otherwise have less access to these important vaccines. The extent to which parents and guardians of children living in poverty are aware of the VFC program or face barriers to participation in it is not known. The Affordable Care Act (ACA) stipulates that ACIP-recommended vaccines be made available to insured children with no copayments or other cost-sharing requirements when administered by an in-network provider. Issues such as proximity to health care providers and clinics, transportation, and convenience of clinic hours also should be investigated and addressed, if needed. Breaks in insurance enrollment (especially Medicaid) are another complication faced by families living below the poverty level that might have a negative effect on their ability to access the medical care system in general (4).

Widespread geographic variation in coverage levels was evident in the ranges of coverage estimates by state and local area, as well as differences by MSA status. Children in more rural (non-MSA) areas had lower coverage with DTaP, poliovirus vaccine, varicella vaccine, $\mathrm{PCV}$, HepA, and rotavirus vaccine than did more urban (MSA central city) children. This also could reflect issues of access and proximity to vaccination providers, including those who administer VFC vaccines.

Evidence-based interventions such as those recommended in The Guide to Community Preventive Services (3) can contribute to addressing the poverty and urbanicity gaps in vaccination coverage. Enhancing access to vaccination services might include conducting home vaccination visits, holding extended office/ clinic hours during the week or on weekends, and establishing vaccination programs in organized child care centers and in settings where the Special Supplemental Nutrition Program for Women, Infants, and Children (WIC) is administered.

Overall, MMR coverage remained within the 92\%-95\% range generally considered adequate for herd immunity for all three diseases (5); however, coverage levels of $<90 \%$ were observed in several states and local areas as well as in the U.S. Virgin Islands. In the era of VFC, the number of cases of

\footnotetext{
†† http://www.cdc.gov/vaccines/programs/vfc/index.html.
}

\section{Summary \\ What is already known about this topic?}

Vaccination has resulted in substantial reductions in morbidity and mortality from childhood diseases in the United States. As new vaccines, such as varicella, pneumococcal conjugate, and rotavirus have been recommended by the Advisory Committee on Immunization Practices, the National Immunization Survey (NIS) has been able to provide important information on vaccine coverage among U.S. children aged 19-35 months and on progress toward meeting coverage targets.

What is added by this report?

The 2015 NIS data reveal no significant changes in overall coverage relative to the previous year. More than $90 \%$ of children were up to date on vaccination against polio; hepatitis $\mathrm{B}$; measles, mumps, and rubella; and varicella. However, children living below the poverty level continued to have lower coverage with rotavirus vaccine; pneumococcal conjugate vaccine; Haemophilus influenzae type b vaccine; and diphtheria, tetanus, and acellular pertussis vaccine. In addition, children living in more rural areas had lower coverage with diphtheria, tetanus, and acellular pertussis vaccine; poliovirus vaccine; varicella vaccine; pneumococcal conjugate vaccine, hepatitis $A$ vaccine; and rotavirus vaccine.

What are the implications for public health practice? CDC will continue to work with state immunization programs through ongoing site visits, teleconferences, and structured interviews to identify reasons for the observed disparities in vaccination coverage by poverty status and to implement effective strategies to eliminate them. Effort is needed to ensure full participation in the Vaccines for Children (VFC) program for eligible children who could benefit from it. Supporting the continued development of state and local immunization information systems to monitor vaccination coverage can help identify areas of undervaccination that might not be easily detected by the national monitoring program. Identifying areas of need and responding with evidence-based interventions will allow continued progress in protecting young children against vaccine-preventable diseases.

measles has dropped substantially, to only 49 in the United States in 2016 (through July 30) (6). Despite high coverage, outbreaks continue to occur, demonstrating that pockets of susceptibility to this highly contagious disease remain. Reductions in rotavirus-related morbidity have been observed in the United States after vaccine introduction, and indirect (herd) effects of vaccination might have contributed to the decline ( 7 ). Additional activities are needed to increase rotavirus coverage as demonstrated by the relatively low overall coverage, large disparity by poverty, and state variation in vaccination rates. Increased rotavirus vaccination coverage is needed to decrease further the overall prevalence of rotavirus gastroenteritis.

The findings in this report are subject to at least three limitations, each of which could lead to bias in estimates 
of vaccination coverage (8). First, response rates were low. Second, the telephone sample frame did not cover the entire target population. Bias from incomplete sample frame and nonresponse may remain after weighting adjustments. Finally, ascertainment of the vaccination status may be incomplete. A total survey error model, including potential underascertainment of vaccinations reported by providers, indicated that some NIS estimates might be too low by about five percentage points (9). However, a recent analysis demonstrated no evidence of substantial change in bias in NIS during the period 1995-2013 (10).

NIS continues to provide valuable national and state level data. In addition, it is important that states continue to obtain local level coverage data useful for identifying pockets of under-vaccinated children. This analysis documents high overall coverage and, importantly, lower coverage in rural and poorer populations. Continued surveillance is needed to monitor coverage, locate pockets of susceptibility, and evaluate the impact of interventions designed to ensure that all children remain adequately protected against vaccinepreventable diseases. More widespread implementation of evidence-based interventions is needed to bring about continued improvement in vaccination coverage among infants and young children in the United States. Financial barriers to vaccine purchase itself are addressed for children living below the poverty level by the VFC program and vaccine-related stipulations in the ACA. Improved access might be achieved through establishment of vaccination programs in child care centers and in WIC settings. Systems-based interventions, such as patient reminder and recall systems, provider reminders, establishment of standing orders for vaccination, and further development of immunization information systems might be useful in improving vaccination coverage among all young children in the United States.

\footnotetext{
${ }^{1}$ Immunization Services Division, National Center for Immunization and Respiratory Diseases, CDC.

Corresponding author: Holly A. Hill, hhill@cdc.gov, 404-639-8044.
}

\section{References}

1. Whitney CG, Zhou F, Singleton J, Schuchat A. Benefits from immunization during the vaccines for children program era-United States, 1994-2013. MMWR Morb Mortal Wkly Rep 2014;63:352-5.

2. Robinson CL; Advisory Committee on Immunization Practices (ACIP), ACIP Child/Adolescent Immunization Work Group. Advisory Committee on Immunization Practices Recommended Immunization Schedules for Persons Aged 0 Through 18 Years-United States, 2016. MMWR Morb Mortal Wkly Rep 2016;65:86-7. http://dx.doi. org/10.15585/mmwr.mm6504a4

3. Community Preventive Services Task Force. The Guide to Community Preventive Services: increasing appropriate vaccination. Atlanta, GA: US Department of Health and Human Services, Community Preventive Services Task Force; 2016. http://www.thecommunityguide.org/ vaccines/index.html

4. Smith PJ, Stevenson J, Chu SY. Associations between childhood vaccination coverage, insurance type, and breaks in health insurance coverage. Pediatrics 2006;117:1972-8.http://dx.doi.org/10.1542/ peds.2005-2414

5. Bednarczyk RA, Orenstein WA, Omer SB. Estimating the number of measles-susceptible children and adolescents in the United States using data from the National Immunization Survey-Teen (NIS-Teen). Am J Epidemiol 2016;184:148-56. http://dx.doi.org/10.1093/aje/kwv320

6. CDC. Notifiable diseases and mortality tables. MMWR Morb Mortal Wkly Rep 2016;65:ND-38.

7. Pollard SL, Malpica-Llanos T, Friberg IK, Fischer-Walker C, Ashraf S, Walker N. Estimating the herd immunity effect of rotavirus vaccine. Vaccine 2015;33:3795-800. http://dx.doi.org/10.1016/j.vaccine.2015.06.064

8. Hill HA, Elam-Evans LD, Yankey D, Singleton JA, Kolasa M. National, state, and selected local area vaccination coverage among children aged 19-35 months_-United States, 2014. MMWR Morb Mortal Wkly Rep 2015;64:889-96. http://dx.doi.org/10.15585/mmwr.mm6433a1

9. Wolter K, Pineau V, Skalland B, et al. Total survey error assessment for socio-demographic subgroups in the 2012 U.S. National Immunization Survey. In: Biemer P, De Leeuw E, Edwards B, Kreuter F, Lyberg L, Tucker C, and West B, eds. Total survey error in practice. Hoboken, NJ: John Wiley \& Sons, Inc. In press 2016.

10. Yankey D, Hill HA, Elam-Evans LD, et al. Estimating change in telephone survey bias in an era of declining response rates and transition to wireless telephones-evidence from the National Immunization Survey (NIS), 1995-2013. Presented at the 2015 American Association for Public Opinion Research (AAPOR) 70th Annual Conference, Hollywood, FL; May 14-17, 2015. 